\title{
Reference values and influence of sex and age on hemogram and clinical biochemistry in protected and endangered Murinsulaner horses
}

\author{
Nikica Prvanović Babić ${ }^{1 *}$, Antun Kostelić ${ }^{2}$, Branimir Novak ${ }^{3}$, \\ Dragica Šalamon², Bruna Tariba ${ }^{2}$, Nino Maćešić1, Tugomir Karadjole ${ }^{1}$, \\ Goran Bačić ${ }^{1}$, and Ljiljana Bedrica ${ }^{4}$
}

${ }^{I}$ Clinic for Obstetrics and Reproduction, Faculty of Veterinary Medicine, University of Zagreb, Zagreb, Croatia

${ }^{2}$ Department for Animal Science, Faculty of Agriculture, University of Zagreb, Zagreb, Croatia

${ }^{3}$ Veterinary station "Bioinstitut", Čakovec, Croatia

${ }^{4}$ Clinic for Internal Deseases, Faculty of Veterinary Medicine, University of Zagreb, Zagreb, Croatia

\begin{abstract}
PRVANOVIĆ BABIĆ, N., A. KOSTELIĆ, B. NOVAK, D. ŠALAMON, B. TARIBA, N. MAĆEŠIĆ, T. KARADJOLE, G. BAČIĆ, LJ. BEDRICA: Reference values and influence of sex and age on hemogram and clinical biochemistry in protected and endangered Murinsulaner horses. Vet. arhiv 89, 25-42, 2019.
\end{abstract}

ABSTRACT

The aim of this study was to establish the exact haematological and clinical biochemistry reference values for horses of the Murinsulaner breed, using the complete population in Croatia $(n=33)$ for the sampling. Haemoglobin concentrations (HB), red blood cell count (RBC), white blood cell count (WBC), packed cell volume $(\mathrm{PCV})$, mean corpuscular volume $(\mathrm{MCV})$, mean corpuscular haemoglobin concentration $(\mathrm{MCHC})$, mean corpuscular haemoglobin $(\mathrm{MCH})$, platelet count and differential white blood cell counts were determined. Clinical biochemistry included the activity of GGT, AST, ALT, CK, ALP, LDH and AMYL, concentrations of glucose (GLUC), total protein (TP), albumin (ALB), cholesterol (CHOL), triglycerides (TRI), calcium (Ca), phosphorus (P), magnesium (Mg), iron (Fe), urea (UREA), creatinine (CREA) and total bilirubin (TBIL). The animals were sorted into groups according to sex and age, and analysis of variance (ANOVA) was preformed using the GLM procedure and Tukey's studentized range test. The results of the two-way ANOVA showed a significantly higher mean for CREA in stallions, for ALB in old horses and CK in young horses. The two-way ANOVA showed significant differences in the means for HB, PCV, and globulins, CK, GLUC, ALB and CREA. The significantly higher means of HB, PCV, GLUC and CREA in the group of old stallions should be further investigated. The influence of age and sex is difficult to interpret due to the critically small population. The reference values for Murinsulaner horses are within ranges for other cold-blooded breeds except TP, Ca and UREA.

Key words: Murinsulaner horse; hemogram; clinical biochemistry; sex; age

\footnotetext{
*Corresponding author:

Assoc. Prof. Nikica Prvanović Babić, PhD, DVM, Clinic for Obstetrics and Reproduction, Faculty of Veterinary Medicine, University of Zagreb, Heinzelova 55, Zagreb, Croatia, Phone: +385 12390 320, Fax: +385 12441 390, E-mail: nikica@vef.hr
} 
N. Prvanović Babić et al.: Hemogram and clinical biochemistry of Murinsulaner horses

\section{Introduction}

Haematological and clinical biochemistry parameters in horses are used as an aid for the clinical diagnosis of organic, infectious and several parasitic diseases. They are also used for monitoring recovery during disease treatment, and to assess the metabolic condition of a single animal or a complete herd (RICKETTS, 1987; LASSEN and SWARDSON, 1995; MESSER, 1995). Haematological data are available for several particular horse breeds, as well as for horses in general (SCHALM et al., 1975; JEFFSCOTT, 1979; EIKMEIER, 1982; HARVEY et al., 1984; ROSE and HODGSON, 1994; PAĐEN et al., 2014). The normal ranges of haematological parameters for healthy individual horses are quite narrow, whereas normal values for a breed fall into a broad range (HODGSON and ROSE, 1994.) The horse is unique in comparison to most other mammalian species in that the spleen is a very capacious organ, storing up to one third of the RBC, and this reserve can be mobilised by exercise, stress or excitement (LASSEN and SWARDSON, 1995; SATUE et al., 2009). The degree of excitement during blood withdrawal depends on the temperament and the breed (RUBIO et al., 1995). For example, according to SATUE et al. (2009) Andalusian horses are less nervous than other warm-blooded breeds, such as Thoroughbreds and Arabians, resulting in several lower haematological reference values for that breed, making diagnosis of slight anaemia difficult in some cases, when dealing with such animals. The same research demonstrated that ageing significantly influences the haematological values of Andalusian broodmares, with the most marked differences in animals older than 13 years, and that these physiological variations must be taken into account in a clinical context, just as the breed of horse itself. Among horses, Thoroughbreds generally have greater RBC counts, lower MCV values and higher total blood volume in relation to body weight than other breeds. In comparison to warmblooded horses, cold-blooded breeds have lower RBC counts, haemoglobin and PCV values, and blood volume. The erythrocyte sedimentation ratio (ESR) is relatively high in cold-blooded animals. The mean N:L ratio in a cold-blooded horse is 1.7:1.0. as compared to 1.0:1.0 in Thoroughbreds and Arabians. American miniature horses have a lower RBC count, as do donkeys and ponies, and an N:L ratio opposite that of full-sized horses (37:59 versus 53:39) as reported by FELDMAN et al. (2000). Similar differences are observed in clinical biochemistry reference values, since TADICH et al. (1997), who determined the clinical biochemistry reference values for draught horses in Chile, observed that four out of nine biochemical parameters differed from the reference values already published by at least $40 \%$.

Although Murinsulaner horses are cold-blooded horses, due to their partial origin based on purebred Arabian and Anglo-Arabian mares (STEINHAUSZ, 1934), they are generally more sensitive and short-tempered than other Croatian cold-blooded breeds (the Croatian Posavina horse and the Croatian Coldblood horse). Furthermore, reference data for Croatian Coldblood and Posavina breed published by PAĐEN et al. (2014) differed significantly from the preliminary report of reference data from hemograms 
for Murinsulaner horses published as a short abstract by PRVANOVIĆ et al. (2010). Therefore, the aim of this study was to determine and explain in detail the reference values for Murinsulaner horses, since there are no published data for haematology and clinical biochemistry for this breed, which is in danger of extinction. Furthermore, our goal was to determine the clinical biochemistry for the observed population of Croatian Murinsulaner horses. Finally, our goal was also to determine the influence of age and sex on both the hemogram and clinical biochemistry. The results obtained should make it possible to establish haematological and biochemical reference values in the blood of Murinsulaner horses that will in turn support clinical diagnosis and further research into horse physiology. Altogether, it will provide a wider basis for the protection and conservation of this important and yet endangered Croatian indigenous horse breed.

\section{Materials and methods}

This study was carried out on the complete population of Murinsulaner horses. Altogether 33 healthy adult Murinsulaner horses (3-17 years old) were divided into four groups according to sex and age; stallions $(\mathrm{n}=5)$, mares $(\mathrm{n}=28)$, and horses younger and older than 7 years $(n=14$ versus $n=19)$. All horses were subjected to a general clinical examination before the taking of blood samples, and in all cases no significant clinical findings were observed. Only animals without evidence of trauma or illness before or during the study were included. None of the mares were pregnant, in order to exclude the influence of pregnancy. All animals belonged to private owners united in the Croatian organisation for breeding and protection of Murinsulaner horses, located around the Međimurje County. Whole blood was collected into $8 \mathrm{~mL}$ EDTA vacutainer tubes and transported to the haematology laboratory within 3 hours of collection. All samples were processed immediately after collection using an IDEXX laserCyte automatic counter, validated for horses. The following parameters were determined: haemoglobin concentration (HB), red blood cell count (RBC), white blood cell count (WBC), packed cell volume (PCV), mean corpuscular volume (MCV), mean corpuscular haemoglobin concentration (MCHC), mean corpuscular haemoglobin $(\mathrm{MCH})$, platelet count and differential white blood cell count (percentage of different WBC subtypes). At the same time, blood was also collected in an SST (serum separator tube) and transferred directly to the laboratory to determine clinical biochemistry. Clinical biochemistry included the activity of the enzymes gamma-glutamyl transferase (GGT), aspartate aminotransferase (AST), alanine aminotransferase (ALT), creatine kinase (CK), alkaline phosphatase (ALP), lactat dehydrogenase (LDH) and amylase (AMYL), and concentrations of glucose (GLUC), total protein (TP), albumin (ALB), cholesterol (CHOL), triglycerides (TRI), calcium $(\mathrm{Ca})$, phosphorus $(\mathrm{P})$, magnesium $(\mathrm{Mg})$, iron $(\mathrm{Fe})$, UREA, creatinine (CREA) and total bilirubin (TBIL). Samples for biochemical analysis were processed using the standard procedures with the biochemical analyser Olympus AU560 (Olympus, Hamburg, Germany). 


\section{N. Prvanović Babić et al.: Hemogram and clinical biochemistry of Murinsulaner horses}

Statistical analysis. The animals were sorted into sex and age groups (21 young mares, seven old mares, three young stallions, two old stallions) and analysis of variance (ANOVA) was performed using the GLM procedure and the Tukey's studentized range test in SAS 9.4 to determine if there were any significant differences between the groups. Unbalanced two-way and one-way ANOVA of sex and age groups were preformed. The procedures MEANS and UNIVARIATE were used to obtain overall and group descriptive statistics.

\section{Results}

Mean values, standard deviation and range for hemogram and clinical biochemistry, together with data obtained from other authors for comparison purposes, are given in Tables 1a, 1b, 2a, 2b, 3a, 3b, 4a and 4b. Tables 1a-b and 2a-b give the differences of the means results of the two-way ANOVA (differences between the old mares, young mares, old stallions and young stallions groups) and one-way ANOVA analyses of sex (stallions versus mares) and age (old versus young animals) groups for the measured hemogram traits and clinical biochemistry of Murinsulaner horses.

There were no significant sex or age differences in the measured hemogram traits of Murinsulaner horses in the one-way ANOVA analyses (Table 1a-b). However, the twoway ANOVA distinguished significantly higher $\mathrm{HB}$ and PCV values in the old stallions in comparison with the young stallions, while the old or young mares did not have significantly different values from one another or the groups of stallions, for these two variables. Additionally, the two-way ANOVA showed old mares to be the group with different globulin (GLOB) values in the lowest part of the range of measurement in this group. However, the differences in the means for the four groups concerning GLOB were not significant, which may be due to the missing observations in the young mare group where nine of the animals lack any results (Table 1a-b).

As shown in Table 2a-b, the one-way ANOVA for sex grouping distinguished differences in the means for CREA, which was higher in stallions. Additionally, the oneway ANOVA for age grouping distinguished differences in the CK means, which were higher in the young group, and the ALB mean value was higher in the old group. This result is further reflected in the two-way ANOVA where a non-significantly lower CK mean was noted for the old stallions, but it must be noted that the values for nine of the young mares are missing. Also, a significantly higher mean value of ALB was found for the old mares, while the old and young stallions did not have significantly different means from each other or the other groups. Moreover, the two-way ANOVA in Table 2a-b shows that the mean value of glucose levels in old stallions is significantly higher than in young stallions, while the old and young mares do not have significantly different means from one another or the groups of stallions.

Differences from other horse breeds and horses in general, considering age and sex, will be explained in the Discussion and are also shown in Tables 3a-b and 4a-b. 
N. Prvanović Babić et al.: Hemogram and clinical biochemistry of Murinsulaner horses

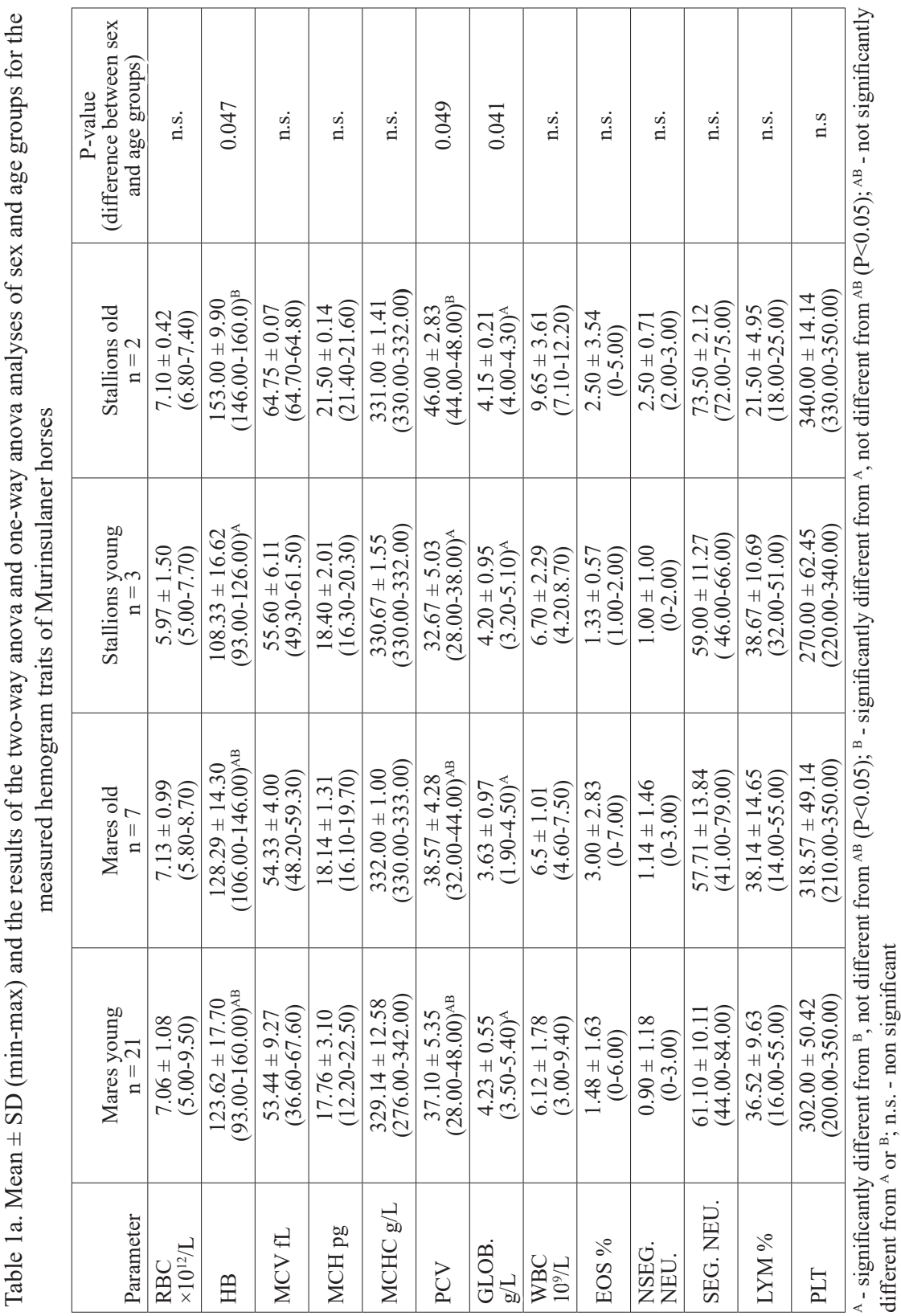

Vet. arhiv 89 (1), 25-42, 2019 
N. Prvanović Babić et al.: Hemogram and clinical biochemistry of Murinsulaner horses

\begin{tabular}{|c|c|c|c|c|c|c|c|c|c|c|c|c|c|}
\hline 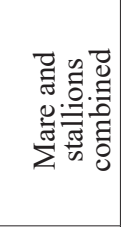 & 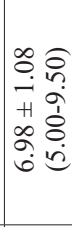 & 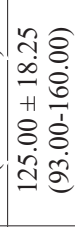 & 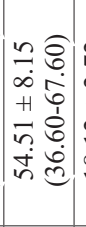 & 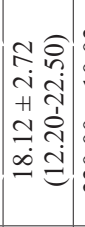 & 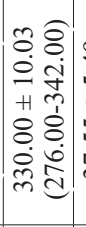 & 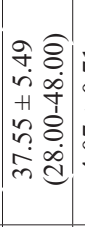 & 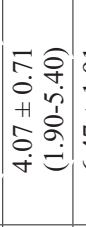 & 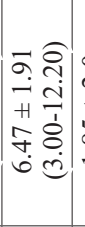 & $\left|\begin{array}{cc}0 & \\
i & \delta \\
+ & \vdots \\
n & i \\
\infty & 1 \\
- & e\end{array}\right|$ & 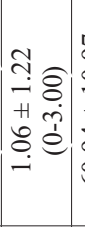 & 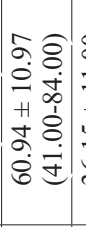 & 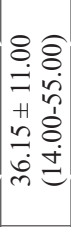 & 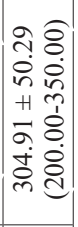 \\
\hline 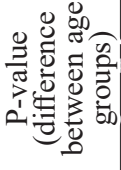 & $\stackrel{\dot{d}}{=}$ & $\stackrel{\dot{g}}{=}$ & $\stackrel{d}{g}$ & $\stackrel{\oplus}{\sharp}$ & 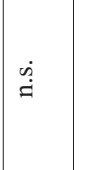 & $\stackrel{\oplus}{\sharp}$ & $\stackrel{\dot{g}}{=}$ & $\stackrel{\dot{\varphi}}{=}$ & $\dot{\mathscr{g}}$ & $\stackrel{\dot{\varphi}}{\sharp}$ & $\stackrel{\dot{\varphi}}{=}$ & $\dot{\mathscr{g}}$ & $\dot{\stackrel{\leftrightarrow}{g}}$ \\
\hline$\vec{\sigma}^{a} \|$ & 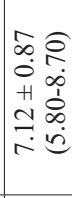 & 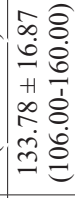 & 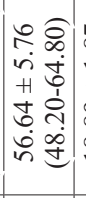 & 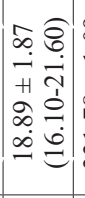 & 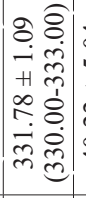 & 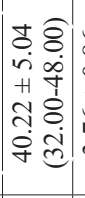 & 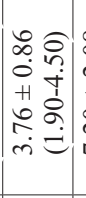 & 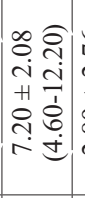 & 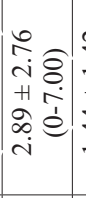 & 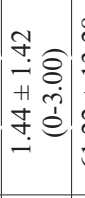 & 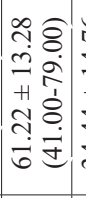 & 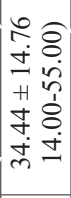 & 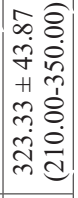 \\
\hline 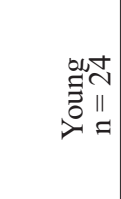 & 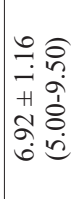 & 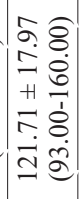 & 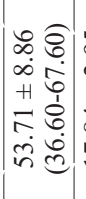 & 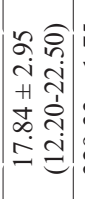 & 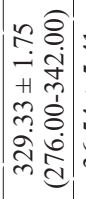 & 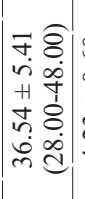 & 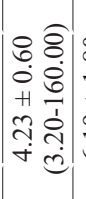 & 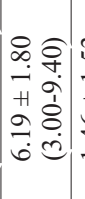 & 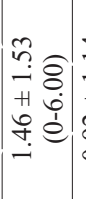 & 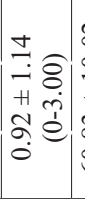 & 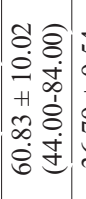 & 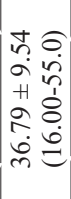 & 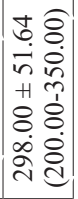 \\
\hline 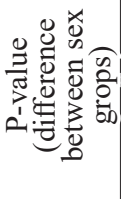 & $\stackrel{\stackrel{\rho}{=}}{=}$ & $\stackrel{\dot{g}}{=}$ & $\stackrel{\leftrightarrow}{=}$ & $\stackrel{\dot{\leftrightarrow}}{=}$ & $\stackrel{\dot{\oplus}}{=}$ & $\stackrel{\dot{\leftrightarrow}}{=}$ & $\stackrel{\dot{g}}{=}$ & $\stackrel{\dot{\leftrightarrow}}{=}$ & $\stackrel{\dot{\leftrightarrow}}{=}$ & $\stackrel{\dot{\leftrightarrow}}{=}$ & $\stackrel{\dot{g}}{=}$ & $\stackrel{\dot{g}}{=}$ & $\stackrel{\dot{g}}{=}$ \\
\hline 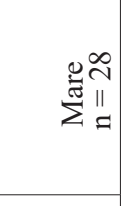 & 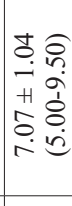 & 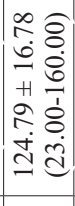 & 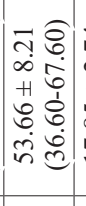 & 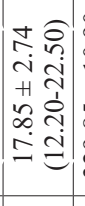 & 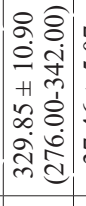 & 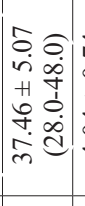 & 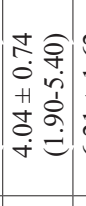 & 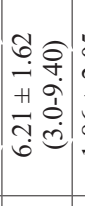 & 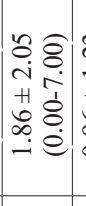 & 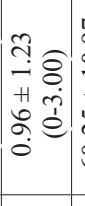 & 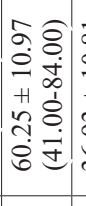 & 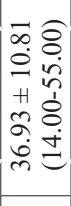 & 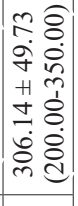 \\
\hline 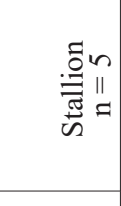 & 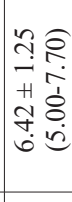 & 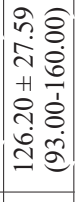 & 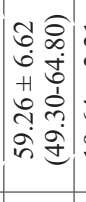 & 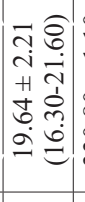 & 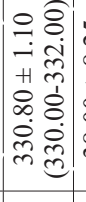 & 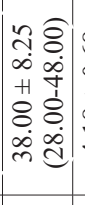 & 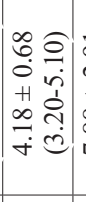 & 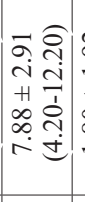 & $\left|\begin{array}{cc}2 & \\
\alpha & \delta \\
+1 & \delta \\
0 & \vdots \\
\infty & e \\
- & \end{array}\right|$ & 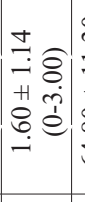 & 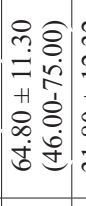 & 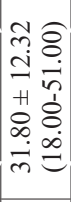 & 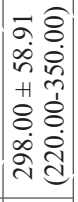 \\
\hline & 定 & $\cong$ & $\begin{array}{l}+1 \\
\dot{\Delta} \\
\dot{\Sigma}\end{array}$ & 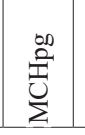 & 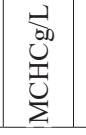 & 己 & 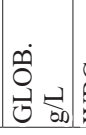 & $\mid \begin{array}{cc}0 & -1 \\
0 \\
3\end{array}$ & $\begin{array}{l}\text { iें } \\
\text { oे }\end{array}$ & 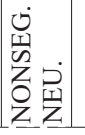 & 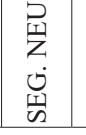 & $\sum_{\lambda}^{\infty}$ & $\vec{a}$ \\
\hline
\end{tabular}


N. Prvanović Babić et al.: Hemogram and clinical biochemistry of Murinsulaner horses

\begin{tabular}{|c|c|c|c|c|c|c|c|c|c|c|c|c|}
\hline 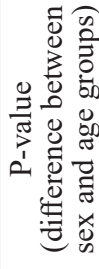 & $\stackrel{\dot{\mathscr{g}}}{\dot{I}}$ & $\stackrel{\dot{g}}{g}$ & ô. & $\stackrel{\dot{\varphi}}{\dot{g}}$ & $\stackrel{\dot{\leftrightarrow}}{=}$ & $\begin{array}{l}\stackrel{0}{0} \\
\stackrel{0}{0}\end{array}$ & $\begin{array}{l}0 \\
8 \\
0\end{array}$ & $\stackrel{\dot{\oplus}}{=}$ & $\stackrel{\dot{\varphi}}{=}$ & $\stackrel{\dot{g}}{=}$ & ठै. & $\stackrel{\dot{g}}{\stackrel{\dot{g}}{ }}$ \\
\hline 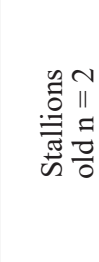 & 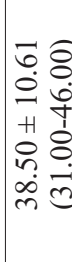 & $\begin{cases}2 & 0 \\
\infty & 8 \\
= & 0 \\
+1 & 8 \\
0 & 8 \\
0 & 0 \\
0 & d \\
m & d\end{cases}$ & 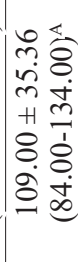 & 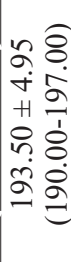 & 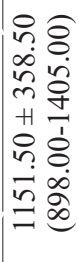 & 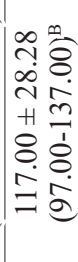 & 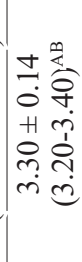 & 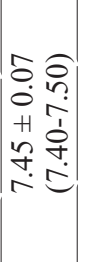 & 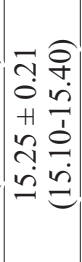 & 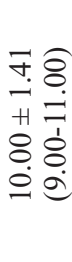 & 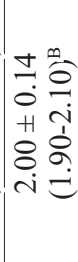 & 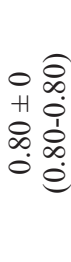 \\
\hline 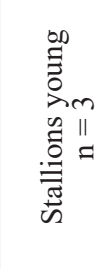 & 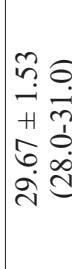 & 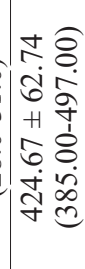 & 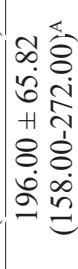 & 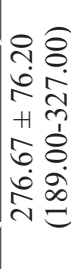 & 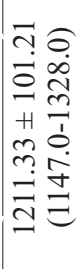 & 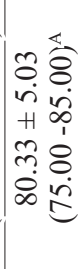 & 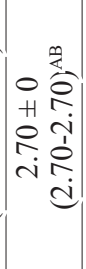 & 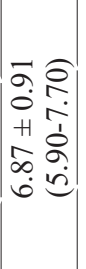 & 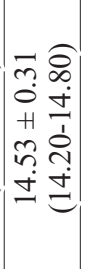 & 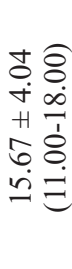 & 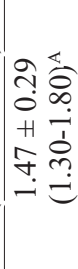 & 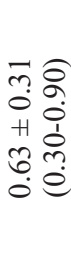 \\
\hline 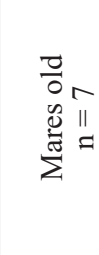 & 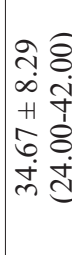 & 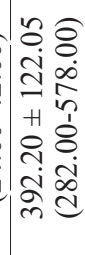 & 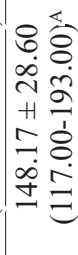 & 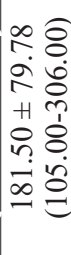 & 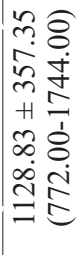 & 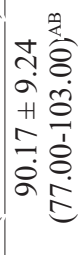 & 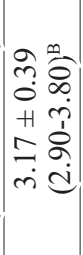 & 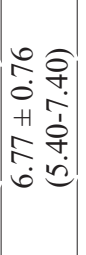 & 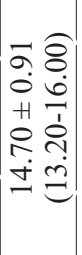 & $\begin{array}{ll} \pm & 0 \\
m & 8 \\
+1 & 0 \\
m & 0 \\
n & 8 \\
0 & 0 \\
0 & =\end{array}$ & 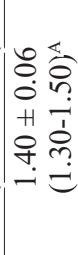 & 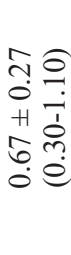 \\
\hline 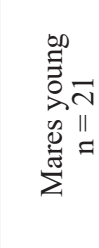 & 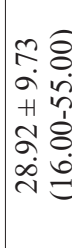 & 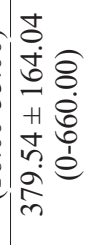 & 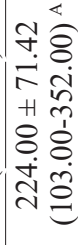 & 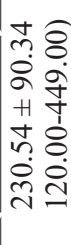 & 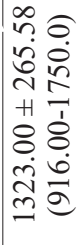 & 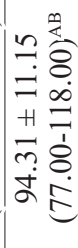 & 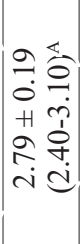 & 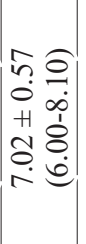 & 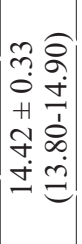 & 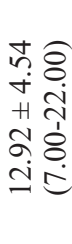 & 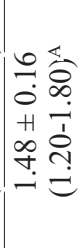 & 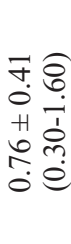 \\
\hline & & 究吉 & 记㝍 & 85 & 鬲寻 & 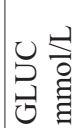 & لم & & 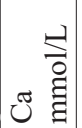 & 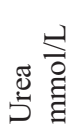 & 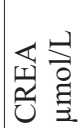 & 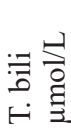 \\
\hline
\end{tabular}

Vet. arhiv 89 (1), 25-42, 2019 
N. Prvanović Babić et al.: Hemogram and clinical biochemistry of Murinsulaner horses

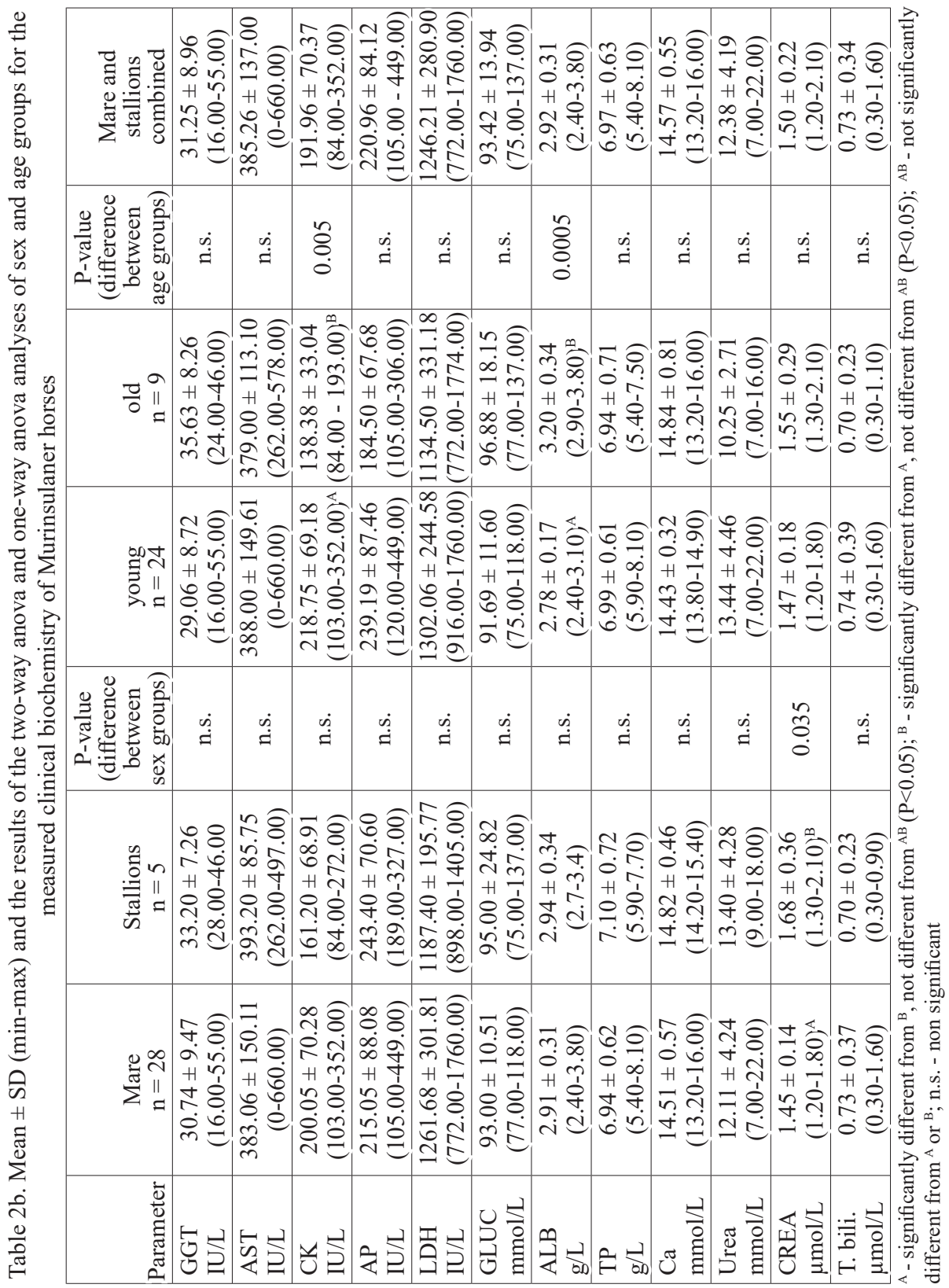


N. Prvanović Babić et al.: Hemogram and clinical biochemistry of Murinsulaner horses

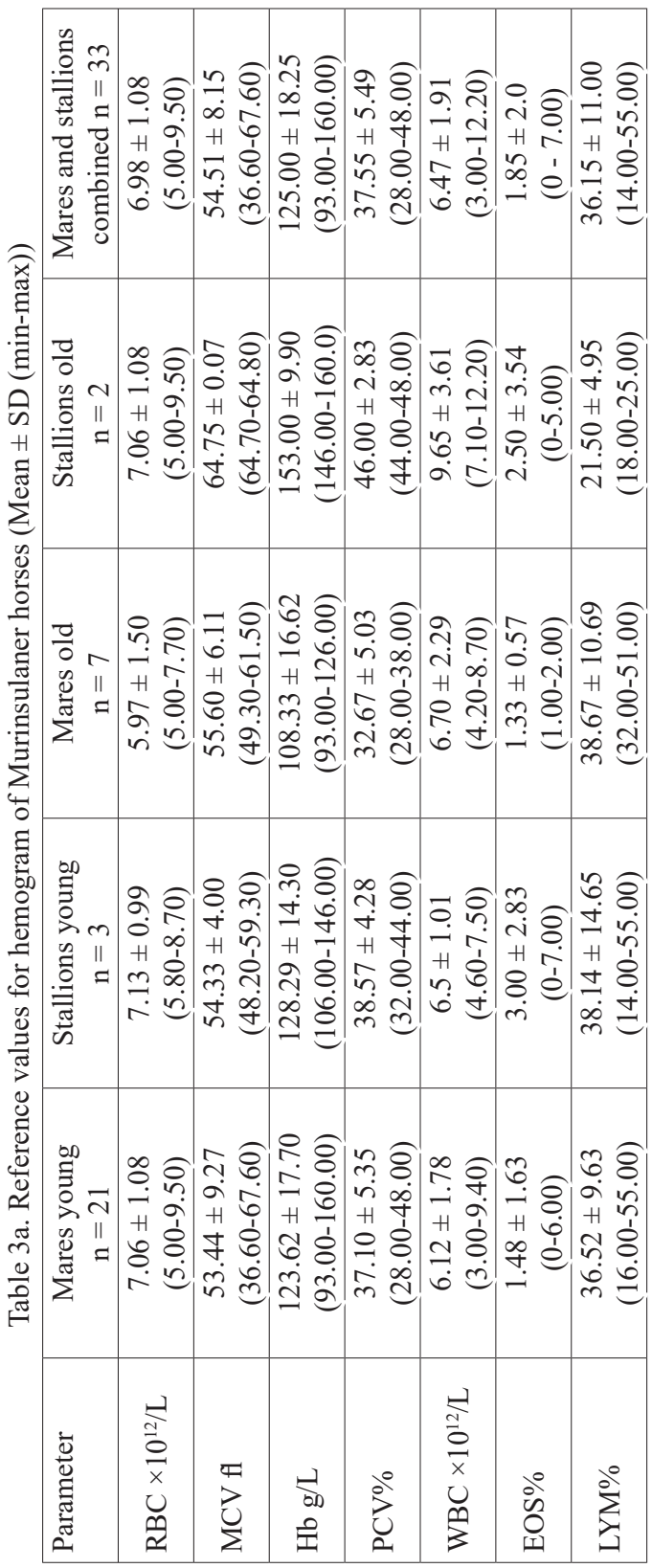

Vet. arhiv 89 (1), 25-42, 2019 
N. Prvanović Babić et al.: Hemogram and clinical biochemistry of Murinsulaner horses

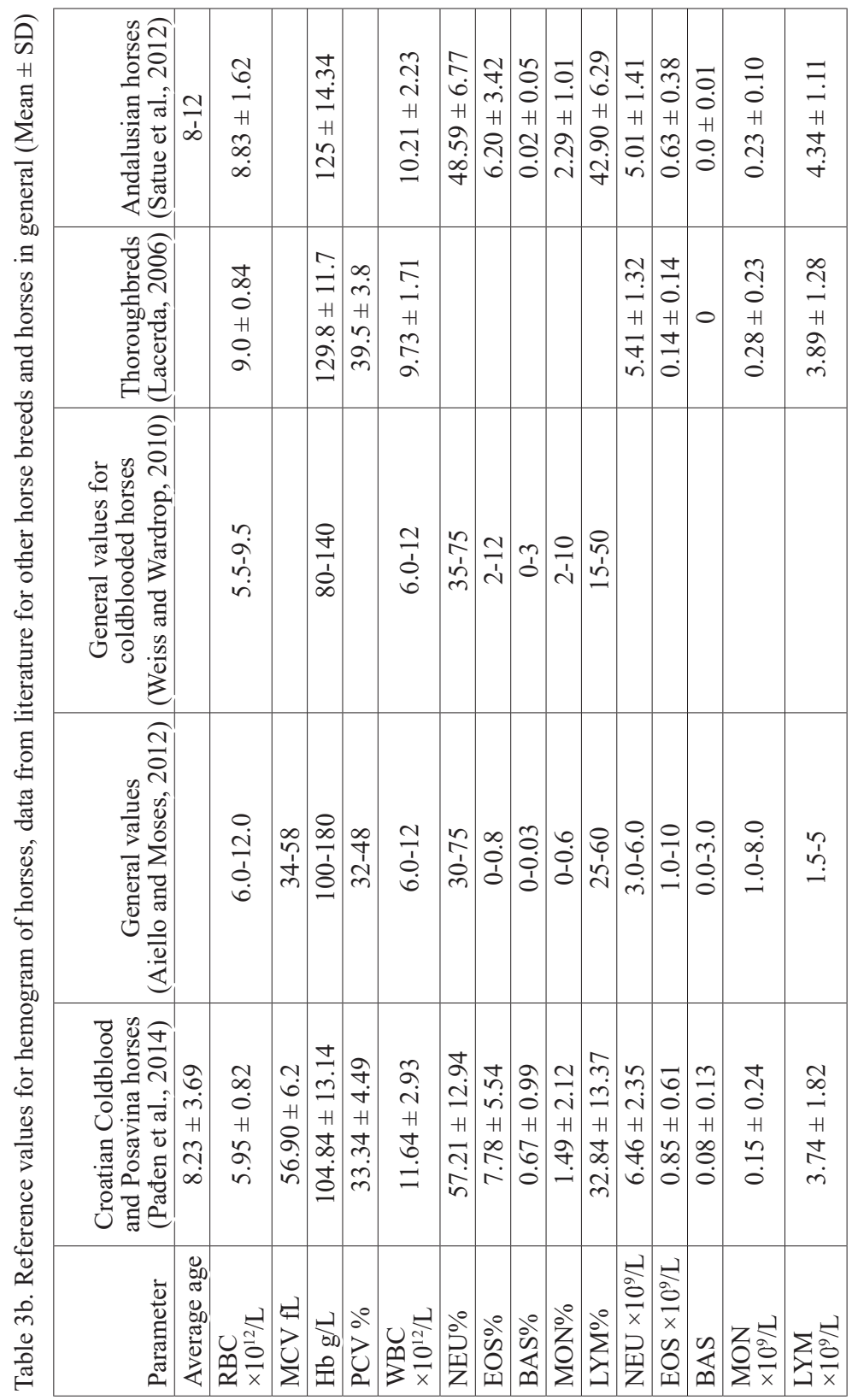


N. Prvanović Babić et al.: Hemogram and clinical biochemistry of Murinsulaner horses

\begin{tabular}{|c|c|c|c|c|c|c|c|c|c|c|c|c|c|c|}
\hline 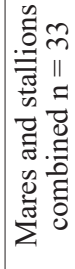 & 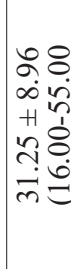 & 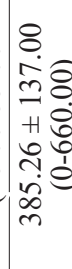 & 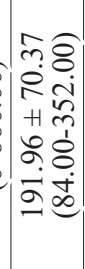 & 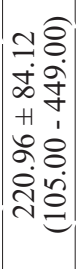 & 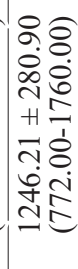 & 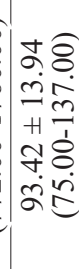 & 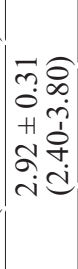 & 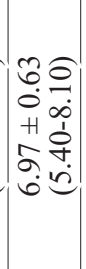 & 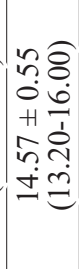 & 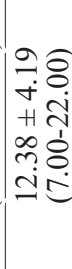 & 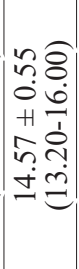 & 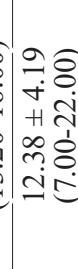 & 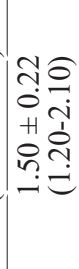 & 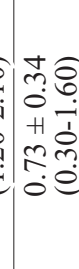 \\
\hline 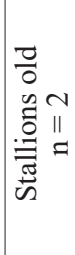 & 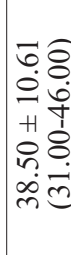 & $\begin{cases}2 & 8 \\
0 & 0 \\
7 & 0 \\
118 \\
8 & 8 \\
0 & 0 \\
m & 0\end{cases}$ & 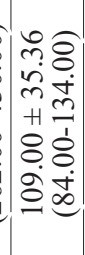 & 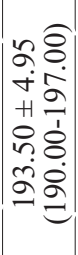 & 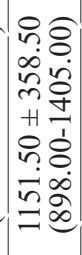 & 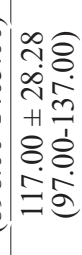 & 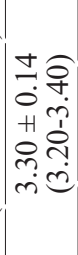 & 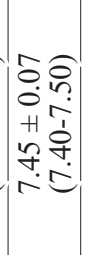 & 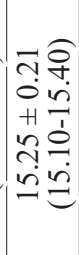 & $\begin{array}{l}78 \\
+1 \\
+1 \\
8 \\
8 \\
0 \\
0\end{array}$ & 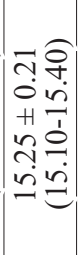 & 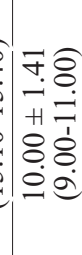 & 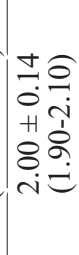 & $\begin{cases}0 & 0 \\
0 & 0 \\
1 & 0 \\
0 & \vdots \\
0 & \vdots \\
0 & \infty \\
0 & 0\end{cases}$ \\
\hline 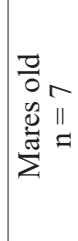 & 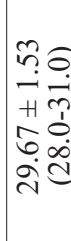 & 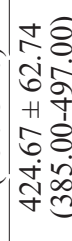 & 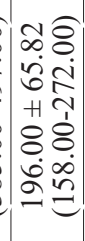 & 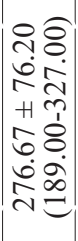 & 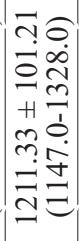 & 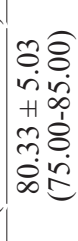 & 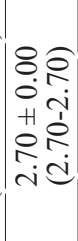 & 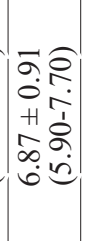 & 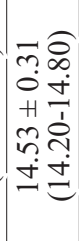 & 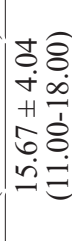 & 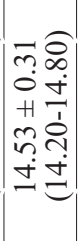 & 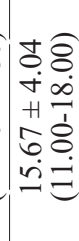 & 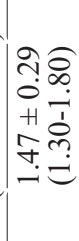 & $\mid \begin{array}{ll}\pi & 0 \\
0 & \vdots \\
1 & 0 \\
0 & 0 \\
6 & 0 \\
0 & 0\end{array}$ \\
\hline 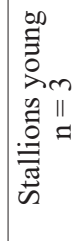 & 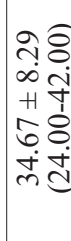 & 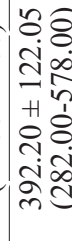 & 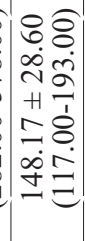 & 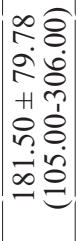 & 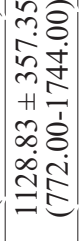 & 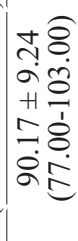 & 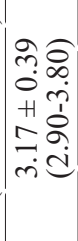 & 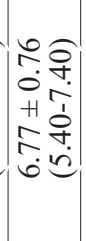 & 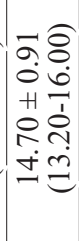 & 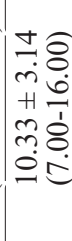 & 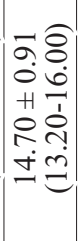 & 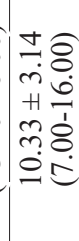 & 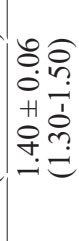 & 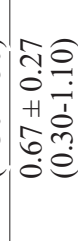 \\
\hline 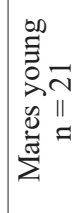 & 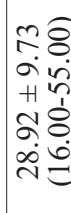 & $\left\{\begin{array}{l}0 \\
0 \\
\dot{0} \\
0 \\
+1 \\
+0 \\
0 \\
0 \\
0 \\
0 \\
0\end{array}\right.$ & 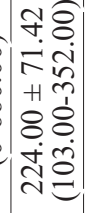 & 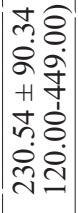 & 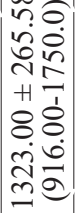 & 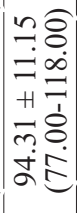 & 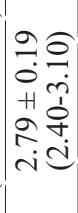 & $\left|\begin{array}{ll}n & 0 \\
0 & 0 \\
+ & 0 \\
+1 & 1 \\
0 & 0 \\
0 & 0\end{array}\right|$ & 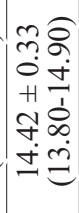 & 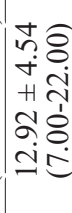 & 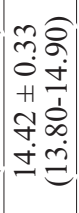 & 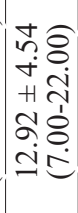 & 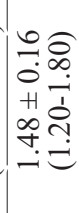 & $\mid \begin{array}{ll}7 & 0 \\
0 & 0 \\
+1 & 1 \\
0 & 0 \\
0 & 0\end{array}$ \\
\hline & & & & $\exists$ & & & $\sum_{\Delta}$ & 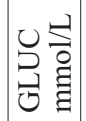 & 坣 & & 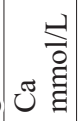 & 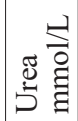 & 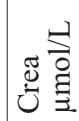 & 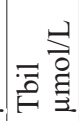 \\
\hline
\end{tabular}

Vet. arhiv 89 (1), 25-42, 2019 
N. Prvanović Babić et al.: Hemogram and clinical biochemistry of Murinsulaner horses

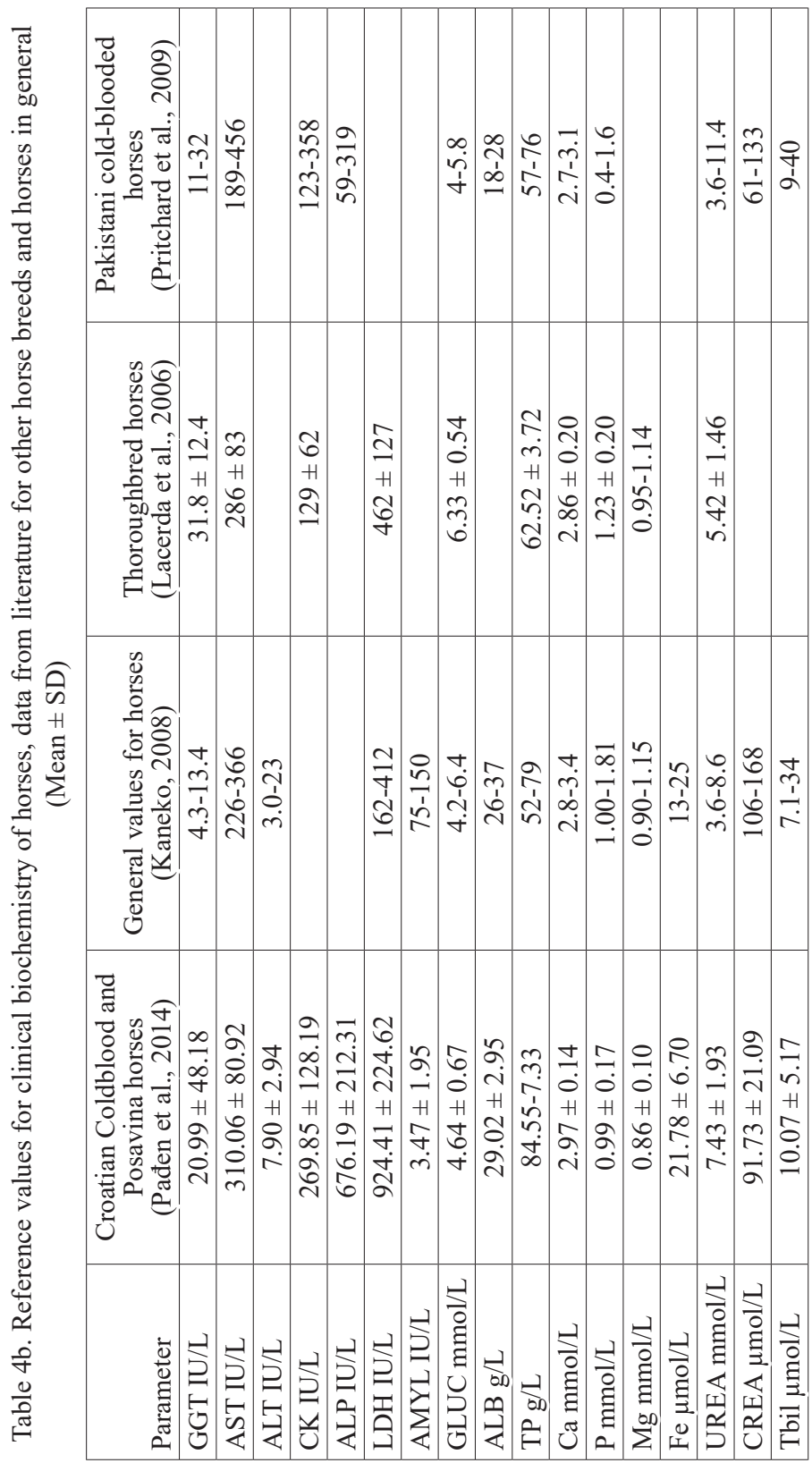




\section{Discussion}

In order to help in preservation and provide better health care of protected and endangered Murinsulaner horses, this study established haematological and clinical biochemistry reference values for the breed. It also took into consideration the influence of sex and age on haematology and clinical biochemistry.

The results obtained, given in Tables 1a, 1b, 2a, 2b, 3a, 3b, 4a, 4b, together with data for horses in general and other particular breeds, showed that the majority of reference values for Murinsulaner horses overlap with the data already published for coldblooded horses, as described by WEISS and WARDROP (2010), and differ from values for Thoroughbreds and Andalusian horses (LACERDA et al. 2006; SATUE et al., 2009). It is interesting to notice that there are some differences compared to the reference values for Posavina and Croatian coldblooded horses reported by PAĐEN et al. (2014). At the same time, the reference values obtained by PAĐEN et al. (2014) overlapped substantially with data reported for Pakistani coldblooded horses by PRITCHARD et al. (2009). Such lower levels of overlapping with our data were unexpected due to the small genetic differentiation between Croatian horse breeds (Posavina, Croatian Coldblood and Murinsulaner as reported by IVANKOVIĆ et al. (2009). It is particularly strange, since Murinsulaner horses are partly kept in stables as working animals, just like the Pakistani working horses, and some are kept outside, just like Croatian Coldblood and Posavina horses, especially mares, in an open system biocenosis of woods and marshy meadows, continuously exposed to different infectious and parasitic diseases, that could also influence the reference haematological values obtained. Since Murinsulaner horses partially originate from Anglo - Arabian mares, which influences their temperament when compared to other cold-blooded breeds (STEINHAUSZ, 1934), further research is needed to determine if this difference could influence the haematology reference values of Murinsulaner horses, as already described for mares of the Carthusian strain by SATUE et al. (2009).

Since some clinical biochemistry referral values for Murinsulaner horses (TP, Ca, UREA) were significantly different than the data already published for horses, further research is needed to determine the reasons for such differences. Similar results were obtained by TADICH et al. (1997), who determined the clinical biochemistry reference values for draught horses in Chile, and observed that four out of nine biochemical parameters differed from the reference values already published by at least $40 \%$.

The statistically higher reference haematology values (HB and PCV) obtained for stallions in our research have also been described by other authors. According to SATUE et al. (2012) the different baseline hemogram values present in stallions when compared with geldings and mares are explained by the probable influence of androgens on erythropoiesis (KELANI and DUROTOYE, 2002). This wasalso described by PAĐEN 
et al. (2014) who determined higher values for haematocrit (PCV) and HB for a group of stallions, compared with mares and geldings. A similar conclusion was drawn by GUPTA et al. (2002) who found higher bilirubin concentrations in stallions than in mares, which is probably related to a higher RBC count. In contrast, HERNANDEZ-GUERRA et al. (2008) found higher total leucocyte count and MCH in Spanish purebred mares. ULUISIK et al. (2013) reported slight differences between male and female thoroughbred foals for PCV and WBC, while TOMENENDALOVA et al. (2014) reported relatively higher HB concentration, leucocyte and monocyte counts in stallions than in Przewalski mares, but without any statistical significance. Similar results are given in the study published by LACERDA et al. (2006) on Thoroughbred horses, who found no significant differences between the sexes.

Since the influence of sex on blood parameters is present and well documented in other mammals, further research is needed to determine sex differences in haematological reference values in horses.

Although the influence of age on hemogram reference values has been documented in various horse breeds by HERNANDEZ-GUERRA et al. (2008), SATUE et al. (2009), GRONDIN and DEWITT (2010) and SGORBINI et al. (2013) there was no significant difference between age groups in our research. ČEBULJ-KADUNC et al. (2002) reported higher erythrocyte count and PCV in 4 month old foals compared to adult mares and stallions of the Lipizzan breed, while ULUISIK et al. (2013) described significant differences in RBC, PCV and MCV in Thoroughbred foals of different ages. SATUE et al. (2009) observed lower total leukocyte and lymphocyte count in pregnant Anadalusian mares older than 13 years. This is consistent with the conclusions given by McFARLANE et al. (2001) and the preliminary report by PRVANOVIĆ et al. (2010) who observed that the decrease in leucocyte count in healthy aged horses may be related to an age associated decrease in immunocompetency. This is in contrast with this study, where no significant difference in leucocyte count was found between age groups.

In our study there was no difference considering age groups for total bilirubin, Urea, $\mathrm{Ca}, \mathrm{AP}, \mathrm{AST}, \mathrm{LDH}, \mathrm{GGT}$, TP, globulin and $\mathrm{MCH}$, but there was a slight difference for CK and albumins. However, in this study significantly higher CK activity was found in mares compared to stallions, and this led to a considerably higher reference interval (Table 2a-b) compared to Thoroughbreds and Pakistani working horses. Similar results were obtained for Posavina and Croatian Coldblood mares by PAĐEN et al. (2014). Since $\mathrm{CK}$ is a sensitive indicator of muscle damage, the explanation given by PAĐEN et al. (2014) is that mares that are kept predominantly outdoors in pasture are more prone to muscle damage than stallions. This is in accordance with our results as well. Furthermore, PAĐEN et al. (2014) found significantly higher albumin concentrations in stallions, that 
is in accordance with the results obtained by GUPTA et al. (2002) while the stallions in our study had slightly lower albumin values than mares.

Similar to AOKI and ISHII (2011), who analysed the clinical biochemistry of heavy draught horses we also did not find any significant changes in Ca between the sexes, while PAĐEN et al. (2014) found significant differences between stallions and mares, which they explained by the difference in pasture components between Posavina and Međimurje, and the potassium oxalate compound in the grass.

\section{Conclusions}

The results obtained provide an insight into metabolism and physiology of endangered and protected Murinsulaner horses. Reference values for Murinsulaner horses are within the range of other cold-blooded breeds. The influence of age and sex is difficult to interpret due to the critically small population. Since the monitored population is small and precious, our reference interval could provide a valuable tool for its preservation and treatment. It should also enable the establishment of hemogram and clinical biochemistry reference values for Murinsulaner horses, as support for clinical diagnosis and further research into horse physiology. Since some clinical biochemistry referral values for Murinsulaner horses (TP, Ca, UREA,) were significantly different than the data already published for horses, further research is needed to determine the reasons for such differences. For more accurate and better results repeated blood sampling is needed, which is doubtful due to the welfare standards for these endangered and protected horses.

\section{References}

AIELLO, S. E., M. A. MOSES (2012): The Merck Veterinary Manual. Sharp \& Dohme Corp., New York, USA.

AOKI, T., M. ISHII (2011): Hematological and biochemical profiles in peripartum mares and neonatal foals (heavy draft horse). J. Equine Vet. Sci. 32, 170-176.

DOI: 10.1016/j.jevs.2011.08.015

ČEBULJ-KADUNC, N., M. BOŽIČ, M. KOSEC, V. CESTNIK (2002): The influence of age and gender on hematological parameter in Lippizan horses. J. Vet. Med. A 49, 217-221.

DOI: 10.1046/j.1439-0442.2002.00439.x

EIKMEIER, H. (1982): Normal values in laboratory diagnosis in the horse. Berl. Munch. Tierarztl Wochenchr. 95, 85-86.

FELDMAN, B. F., J. G. ZINKL, N. C. JAIN (2000): Schalm's Veterinary Haematology. $5^{\text {th }}$ ed., Lippincott Williams \& Wilkins, Philadelphia

GRONDIN, T. M., S. F. DEWITT (2010): Normal haematology of the horse and donkey. In: Schalm's Veterinary Haematology. (Weiss, D. J., K. J. Wardrop, Eds.) Wiley Blackwell Inc., Ames, IA, pp. 821-828. 
N. Prvanović Babić et al.: Hemogram and clinical biochemistry of Murinsulaner horses

GUPTA, A. K, S. KUMAR, Y. PAL (2002): Biochemical, haematological and thyroid hormone profile in healthy Indian Kathiawari horses. Asian Austral. J. Anim. 15, 1215-1221.

DOI: 10.5713/ajas.2002.1215

HARVEY, R. B., M. B. HAMBRIGHT, L. D. ROWE (1984): Clinical, biochemical and hematologic values of the American miniature horse: reference values. Am. J. Vet. Res. 45, 97-101.

HERNANDEZ-GUERRA, A., M. SATUE, K. LORENTE, C. GARCES (2008): The influence of age and gender on haematologic parameters in andalusian horses. Proceedings of XIV Veterinary European Equine Meeting, Venice, Italy, pp. 400-402.

HODGSON, D. R., R. J. ROSE (1994): The Athletic Horse: Principles and Practice of Equine Sports Medicine. Saunders, Philadelphia.

IVANKOVIĆ, A., J. RAMLJAK, P. DOVČ, N. KELAVA, M. KONJAČIĆ (2009): Genetic structure of three Croatian horse breeds: implications for their conservation strategy. Ital. J. Anim. Sci. 8, 677-689.

DOI: $10.4081 /$ ijas.2009.677

JEFFSCOTT, L. B. (1979): The diagnostic value of hematological and clinical chemical test in equine practice. Vet. Ann. 19, 115-125.

KELANI, O. L., L. A. DUROTOYE (2002): Haematological responses of the African giant rat (Cricetomys gambianus) to castration and androgen replacement.Vet. arhiv 72, 39-49.

KANEKO, J. J., J. W. HARVEY, M. L. BRUSS (2008): Clinical Biochemistry of Domestic Animals. $6^{\text {th }}$ ed., Academic Press, San Diego, California, USA, pp. 904.

LACERDA, L., R. CAMPOS, M. SPERB, E. SOARES, P. BARBOSA, E. GODINHO, R. FERREIRA, V. SANTOS, F. D. GONZALEZ (2006): Hematologic and biochemical parameters in three high performance horse breeds from Southern Brazil. Arch. Vet. Sci. 11, 40-44 (in Spanish).

LASSEN, E. D., C. J. SWARDSON (1995): Hematology and hemostasis in the horse: normal functions and common abnormalities. Vet. Clin. N. Am.-Equine 11, 351-389.

McFARLANE, D., D. C. SELLON, S. A. GIBBS (2001): Age related quantitative alterations in lymphocyte subsets and immunoglobulin isotypes in healthy horses. Am. J. Vet. Res. 62, 14131417.

DOI: 10.2460/ajvr.2001.62.1413

MESSER, N. T. (1995): The use of laboratory test in equine practice. Vet. Clin. N. Am.-Equine 11, 345-350.

DOI: 10.1016/S0749-0739(17)30305-X

PAĐEN, L., T. GOMERČIĆ, M. ĐURAS, H. ARBANASIĆ, A. GALOV (2014): Hematological and serum biochemical reference values for the Posavina and Croatian Coldblood horse breeds. Acta Vet. Beograd 64, 200-212.

DOI: $10.2478 /$ acve-2014-0019 
N. Prvanović Babić et al.: Hemogram and clinical biochemistry of Murinsulaner horses

PRITCHARD, J. C., C. C. BURN, A. R. S. BARR, H. R. WHAY (2009): Haematological and serum biochemical reference values for apparently healthy working horses in Pakistan. Res. Vet. Sci. 87, 389-395.

DOI: 10.1016/j.rvsc.2009.05.003

PRVANOVIĆ, N., A. KOSTELIĆ, B. NOVAK, M. CERGOLJ, J. GRIZELJ, M. SAMARDŽIJA, N. FILIPOVIĆ, M. LIPAR, T. DOBRANIĆ (2010): The influence of age and gender on haematological parameters in protected and endangered Murinsulaner horses. Proceedings of the XVI ${ }^{\text {th }}$ SIVE International Congress, Bologna, Italy, pp. 30-31.

RICKETTS, S. W. (1987): The laboratory as an aid to clinical disorders. Vet. Clin. N. Am.-Equine 3, 445-460.

DOI: $10.1016 / \mathrm{S} 0749-0739(17) 30658-2$

ROSE, R. J., D. R. HODGSON (1994): Hematology and biochemistry. In: The Athletic Horse: Principles and Practice of Equine Sports Medicine. (Hodgson, D. R., R. J. Rose, Eds.), W. B. Saunders, Philadelphia, pp. 63-76.

RUBiO, M. D., A. MUNDOZ, R. SANTISTEBAN, P. TOVAR, F. M. CASTEJON (1995): Comparative haematological study of two breeds of foals (Andalusian and Arab) subjected to exercise of progressive intensity. J. Vet. Med. Sci. 57, 311-315.

DOI: $10.1292 /$ jvms.57.311

SATUE, K., O. BLANCO, A. MUNOZ (2009): Age related differences in the hematological profile of Andalusian broodmares of Carthusian strain. Vet. Med-Czech 54, 175-182.

DOI: $10.17221 / 32 / 2009$-vetmed

SATUE, K., A. HERNANDEZ, A. MUNOZ (2012): Physiological factors in the interpretation of equine hematologic profile. In: Hematology - Science and Practice. (Lawrie, C. H, Ed.), InTech., pp. 577.

SCHALM, O. W., N. C. JAIN, E. J. CAROLL (1975): Veterinary Hematology. Lea \& Febiger, Philadelphia.

SGORBINI, M., F. BONELLI, A. ROTA, P. BARAGLI, V. MARCHETTI, M. CORRAZZA (2013): Haematology and clinical chemistry in Amiata donkey foals from birth to 2 months of age. J. Equine Vet. Sci. 33, 35-39.

DOI: 10.1016/j.jevs.2012.04.010

STEINHAUSZ, M. (1934): Breeding horses in Međimurje. Poljoprivredna knjižnica, Zagreb (in Croatian).

TADICH, N., G. MENDEZ, F. WITTWER, K. MEYER (1997): Blood biochemical values of load cart draught horses in the city of Valdivia (Chile). Arch. Med. Vet. 29, 45-53.

TOMENENENDALOVA, J., R. VODICKA, I. UHRIKOVA, J. DOUBEK (2014) Determination of haematological and biochemical parameters of Przewalski horses (Equus Przewalskii) kept by the Prague Zoo. Vet. Med-Czech 59, 11-21.

DOI: 10.17221/7242-VETMED

Vet. arhiv 89 (1), 25-42, 2019 
N. Prvanović Babić et al.: Hemogram and clinical biochemistry of Murinsulaner horses

ULUISIK, D., E. KESKIN, T. OZAYDIN (2013): Age and gender related changes in hematological parameters of thoroughbred foals. Biotech. Histochem. 88, 345-349.

DOI: $10.3109 / 10520295.2013 .788213$

WEISS, D. J., K. J. WARDROP (2010): Schalm's Veterinary Hematology. $6^{\text {th }}$ ed., Wiley Blackwell, Ames, Iowa, USA, p. 1206.

Received: 14 September 2018

Accepted: 5 November 2018

\section{PRVANOVIĆ BABIĆ, N., A. KOSTELIĆ, B. NOVAK, D. ŠALAMON, B. TARIBA, N. MAĆES̆IĆ, T. KARADJOLE, G. BAČIĆ, LJ. BEDRICA: Referentne vrijednosti i utjecaj spola i dobi na hemogram i kliničku biokemiju zaštićene i ugrožene pasmine međimurski konj. Vet. arhiv 89, 25-42, 2019.}

\section{SAŽETAK}

Svrha ovoga rada bila je utvrditi referentne vrijednosti hematologije i kliničke biokemije za konje pasmine međimurski konj, pri čemu je korištena cjelokupna preostala populacija $(n=33)$. Također se želio utvrditi utjecaj dobi i spola na vrijednosti krvne slike i kliničke biokemije. Dobiveni rezultati jesu referentne vrijednosti za pasminu međimurski konj. Također upućuju na razlike među spolovima za hematokrit i hemoglobin. Nije bilo razlike među dobnim skupinama za ukupni bilirubin, ureju, Ca, AP, AST, LDH, GGT, TP, globulin i MCH, iako je postojala neznatna razlika za CK i albumine. Referentne vrijednosti za konje pasmine međimurski konj uklapaju se u već objavljene referentne vrijednosti za ostale pasmine hladnokrvnih konja, iako od njih neznatno odstupaju. Znatno odstupaju TP, Ca i ureja. Potrebna su dodatna istraživanja kako bi se utvrdilo radi li se o pasminskom svojstvu ili o nečemu drugom. Bilo bi korisno provesti niz mjerenja s ponavljanjem uzorkovanja krvi, kako bi se točno utvrdilo o čemu se radi.

Ključne riječi: međimurski konj; klinička biokemija; hemogram; dob; spol 